\title{
Numerical Study of Drying of Suspension Droplets in High Rate Evaporation Processes: Onset of Buckling
}

\author{
Seyyed Morteza Javid ${ }^{1}$, Christian Moreau ${ }^{2}$, Javad Mostaghimi ${ }^{3 *}$ \\ ${ }^{1}$ Department of Mechanical and Industrial Engineering, University of Toronto, Toronto, Canada \\ ${ }^{2}$ Department of Mechanical, Industrial and Aerospace Engineering, Concordia University, Montreal, Canada \\ ${ }^{3}$ Department of Mechanical and Industrial Engineering, University of Toronto, Toronto, Canada \\ *mostag@mie.utoronto.ca
}

\begin{abstract}
A three-dimensional model based on Fast Interface Particle Interaction (FIPI) is developed for drying a single suspension droplet in processes with a high evaporation rate. The numerical model is able to describe consecutive states of the agglomeration process including droplet shrinkage, particles accumulation on droplet surface, and buckling. Also, the three-dimensional analysis sheds more light on the complex phenomena of buckling specially interaction of droplet interface and suspended solid particles. The model uses a Lagrangian approach for tracking suspended solid particles and Eulerian approach for the liquid phase. It is found that particle-induced surface pressure drives the buckling of suspension droplet. It is shown that the particleinduced surface pressure increases during evaporation until it reaches a threshold value at the onset of buckling. When the surface pressure is larger than surface tension, deformation of droplet originates in locations with lower concentration of particles.
\end{abstract}

\section{Keywords- Buckling, Suspension Droplet, Level Set, Evaporation}

\section{INTRODUCTION}

Drying of suspension droplet appears in many applications such as interfacial materials [1-4], biological systems [5, 6] and industrial processes $[7,8]$. Theoretical description of this phenomenon dates back to the early nineteenth century [9]. However, accurate numerical modeling of such analytical description poses a great challenge due to discontinuity in material properties, complex boundaries and need the simultaneous solution of the equations for solid and liquid phases. For simulation of drying of suspension droplet, the following equations should be solved: governing flow equations; solid particles equation of motion; and equations for tracking droplet interface. Solving these set of equations requires a suitable and consistent numerical scheme which is not only computationally effective but also be able to accurately capture fluid/solid interface and corresponding forces. The development of accurate and efficient computational methods to describe solid particles behavior suspended in drying suspension droplets is currently the subject of intense research effort [10-16].
One perhaps can distinguish three major numerical methods for this phenomenon: Lagrangian-Lagrangian, EulerianLagrangian and Eulerian-Eulerian. In the LagrangianLagrangian method, both fluid and solid treats as particles. Each phase has separated respective domain discretization and numerical scheme and the interface is modeled using a fitted unstructured grid [17] so that the Lagrangian-Lagrangian method can be used for complex moving boundary problems [18]. Another advantage of these methods is reduction in storage and calculation which makes it best candidate for application in real-time simulations, such as animation and gaming [19]. However, inaccurate capturing of discontinuities [20], inadequate prediction of forces exchange between the fluid and solid [21], the restriction of spatial resolution setting [22] and the lack of temporal convergence [17] are the major disadvantages of Lagrangian-Lagrangian method. Smoothed Particle Hydrodynamics (SPH) [23] and Moving Particle SemiImplicit (MPS) [24] can be recognized as example of this class. Regarding the Eulerian-Eulerian approach, mass and momentum equations for each phase, solid and liquid, are solved in a fixed mesh. Each cell contains certain fractions of liquid and solid in such a way that the total volume fractions of phases sum to unity [25]. The Eulerian-Eulerian is suitable for any application with high concentration of the particle phase [26]. Also, this method requires relatively low computational cost for one additional set of equations [27]. However, this approach needs considerable modelling efforts since trajectory and averaging of solid particles are not performed at a computational level and are implicitly calculated at a hypothetical level [28]. It should also be mention that diffusion and heat transfer coefficients are not accurate in the EulerianEulerian approach and there is some uncertainty over the calculated coefficients [27].

The Eulerian-Lagrangian approach has the merits of both previously mentioned numerical methods. It not only can accurately capture the droplet interface and forces exchange between the fluid and solid but also has computationally cheap solutions. In this method, the entire computational domain is occupied with fluid including both gas and liquid phases. The solid particle, however, is treating as a mass point in the fluid mesh and it is tracked using accurate constitutive equations including equation for calculating the fluid/solid interactions. There are several phase interface tracking schemes for fixed grid flow solvers, among them the marker method, the volume- 
of-fluid (VOF) method and the level set method. Each of these tracking methods has it is strengths and weaknesses, such that no clear gold-standard has emerged that is applicable to the wide range of possible multiphase flow phenomena [29-32]. However, existence of spurious current in in the interface using the VOF technique as well as robustness and efficiency of the level set makes the level set method the best candidate for evaporation-driven solid particles agglomeration processes like drying of suspension droplet [33-35].

The purpose of this study is to develop a EulerianLagrangian approach based on FIPI to accurately simulate successive stages of drying of a single droplet in high rate evaporation processes. To minimize numerical errors imposed by spurious currents at the interface, the level set technique is used for tracking the droplet interface. For tracking solid particles, equation of motion for particles is solved, and suitable and consistent numerical schemes are designed to accurately implement the exerted forces. The numerical results reveal that particle-induced surface pressure plays an important role in drying of suspension droplet. It is shown that shrinkage of drying suspension droplet continues until the particleinduced surface pressure overcomes surface tension. Then, droplet will buckle at locations with lower concentration of particles.

\section{GOVERNING EQUATIONS}

A three-dimensional model based on FIPI method [34] is developed to describe the drying of suspension droplet. FIPI is a Eulerian-Lagrangian approach using to model the fluid flow, solid particles dynamics and their interaction in complex multiphase flows. The computational domain is a cubic box of $L=20 \pi \mu m$ discretized using a uniform mesh. It should be mention that the numerical method can only be used in periodic domain on a uniform structured grid. However, this restriction is not an issue for simulation of evaporation of suspension droplet. A mesh refinement study was performed in which the mesh size was gradually reduced until no significant changes were seen in the simulation results $\left(n_{x}=n_{y}=n_{z}=75\right.$ ). The continuity, momentum, and level set equations are numerically solved by the 3D standard Fourier-spectral method to obtain the velocity and droplet spatial distributions throughout the domain [36].

Considering the flow to be incompressible, laminar and Newtonian, the governing equations of each phase solved for low-Reynolds number cases in which fluid inertia is negligible will become:

$$
\nabla \cdot u=0
$$

$$
-\nabla p+\mu \nabla^{2} u+\xi \nabla \phi+\sum_{i=1}^{N} F_{i} \delta\left(x-x_{i}\right)=0
$$

where $\xi \nabla \phi$ is the volumetric force acting on the fluid due to surface tension. The summation term presents all the forces exerted on the droplet by the ${ }^{N}$ particles. This term includes delta forces of strength $F_{i}$ applied at the center $x_{i}$ of each particle. $F_{i}$ is comprised of the drag force $\left(F_{D}\right)$ and the interface-particle capillary force $\left(F_{P}\right)$ [34]. These sets of equations are numerically solved to obtain the velocity $(u)$ and pressure $(P)$ in the droplet. In this method, the interface is traced by the level set function $\phi$ which for a domain $\omega$ (Fig.1) is defined as follow:

$$
\phi(\vec{x}, \mathrm{t})=\left\{\begin{array}{cc}
<0 & \vec{x} \in \omega^{-} \\
0 & \vec{x} \in \partial \omega \\
>0 & \vec{x} \in \omega^{+}
\end{array}\right.
$$

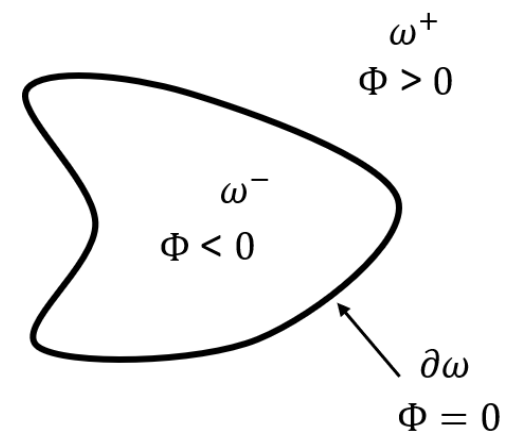

Fig.1 A representation of the level set function for a numerical domain

The advection of the level set function through the computational domain is calculated according to Cahn-Hilliard equation [34]:

$$
\frac{\partial \phi}{\partial t}+u \cdot \nabla \phi=M \nabla^{2} \xi
$$

where $\xi$ is the chemical potential which is derivative of total free energy density $\left(E_{s}\right)$ with respect to the level set function:

$\xi=\frac{\partial E_{s}}{\partial \phi}$

$E_{s}$ is the free energy of system including bulk free energy and interfacial energy [37]:

$$
E_{s}=\frac{\lambda}{4 \varepsilon^{2}}\left(\phi^{2}-1\right)^{2}+\frac{1}{2} \lambda|\nabla \phi|^{2}
$$


where $\lambda=\frac{3}{2 \sqrt{2}} \gamma \varepsilon$ is a numerical parameter proportional to the surface tension $(Y)$ and the thickness of the fluid interface ( $\varepsilon$ ), and $M$ is a mobility parameter.

In this study, instead of solving the evaporation model, evaporation of droplet is simulated by decreasing the size of droplet based on the so-called "D2-law". This treatment enables the uniform decrease in the size of suspension droplet. The applied shrinkage of the droplet size is determined based on the rate of evaporation and operating conditions.

By neglecting particles inertia, particles velocity can be obtained using a balance between forces acting on the particles:

$F_{n}+F_{c}+F_{p}=0$

where $F_{D}, F_{C}$ and $F_{P}$ are drag force, force on particle due to particle-particle interaction and interface-particle capillary force, respectively.

The drag force $\left(F_{D}\right)$ exerted on particles is given by:

$F_{D}=6 \pi \mu r_{p}\left(U_{P}-u\right)$

where $U_{p}, \mu$ and $r_{p}$ are particle velocity, liquid viscosity, and particle radius, respectively.

Various models have been developed to model the particleparticle interaction forces. Linear models are the most intuitive and stable models [38]. In this study, a linear model is used to consider the particle-particle interaction forces [39]:

$F_{c}^{\alpha \beta}=\left\{\begin{array}{cc}-k\left(r-r_{c}\right) n_{\alpha \beta} & r<r_{\epsilon} \\ 0 & r>r_{r}\end{array}\right.$

where $\mathrm{k}_{,}, r_{\epsilon}$, and $n_{\kappa \beta}$ are stiffness parameter, particles distance, cut-off length $\left(r_{c}=5 r_{p}\right)$, and the unit vector along the line connecting centers of particles $\alpha$ and $\beta$ [40].

The main driving force of the agglomeration process is interface-particle capillary force resulting from surface tension. This force only exerts on the particles locating at the droplet interface [34]:

$F_{p}= \begin{cases}\kappa \pi \gamma d n & \left|\phi\left(x_{\alpha^{\prime}} t\right)\right| \leq \phi^{*} \\ 0 & \left|\phi\left(x_{\kappa_{0}} t\right)\right| \geq \phi^{*}\end{cases}$

where ${ }^{\kappa}, d, n$, and $\phi^{*}$ are non-dimensional parameter characterizing the effect of capillary forces, minimum distance between the particle center and the zero level set, the unit normal vector from the interface to the particle center, and maximum distance for which the particle desorbs from the fluid interface $\left(\phi^{*}=r_{p}\right) .{ }^{K}$ can be approximately determined by calculating the work of desorption of $F_{P}$ and equating the result to single-particle adsorption energy expressions [34]:

$$
\kappa=2(1-|\cos \theta|)^{2}
$$

where $\theta$ is the contact angle of solid particles accumulated on the droplet interface (Fig.2).

Since the size of dispersed particles is far smaller than the liquid droplet, the effect of curvature in the liquid- particle interfacial region is negligible and the contact angle can be assumed to be constant throughout the process [41], the contact angle is equal to $90^{\circ}$ in this study.

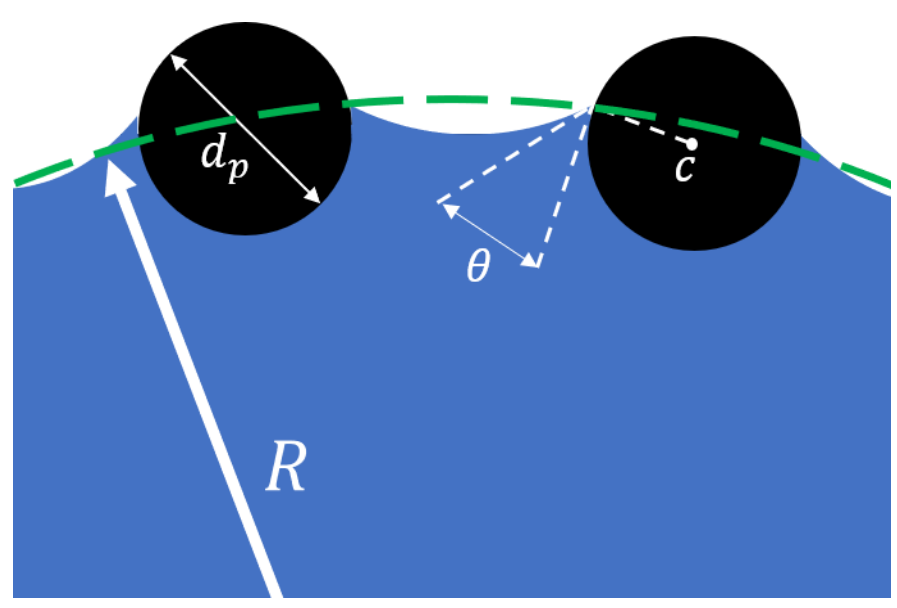

Fig.2 Schematic illustration of the contact angle

\section{RESULTS AND DISCUSSSION}

Fig.3 displays the snapshots of evaporation of a stationary suspension droplet in which monodisperse spherical particles are uniformly dispersed in the liquid phase. For evaporation of droplet, the rate of evaporation is calculated based on the operating condition. Further details on the simulation and properties of suspension droplet are given in Table 1 and Table 2 , respectively. The 3D numerical results are better seen with a cross section showing the solid particles inside the droplet along with the total free energy of the droplet. The corresponding time measured from the beginning of evaporation is given at the left of each image.

The evaporation of liquid phase gradually shrinks the suspension droplet. During isotropic decrease of the droplet diameter (Fig.4), the surface tension force accumulates the solid particle on the droplet interface. Since the rate of evaporation is considerably larger that particle diffusivity inside the liquid phase, particles do not have enough time to move into the center of droplet and forms a packed spherical particle aggregate on the droplet surface $\left(P e=K_{/} / 8 D_{p l} \gg 1\right)$. The particle accumulation on the droplet surface leads to formation of a viscous shell which can alter the behavior of a 
drying droplet. The energy of shell comes from the interaction forces between neighbor particles. The energy of shell gradually increases due to increase of number of particles packed in the shell and reduction of distance between particle during the shrinkage. The energy of shell is expressed as follows [36]:

$$
E_{p}=\sum_{k>l} F_{i}^{k l} l_{i}^{k l}
$$

where $F_{i}^{k l}$ and $l_{i}^{k l}$ are the modulus of the interparticle force between particle $\mathrm{k}$ and particle $\mathrm{l}$ and the corresponding interparticle separation, respectively.

The pressure difference between inside and outside of droplet can be calculated using Young-Laplace equation:

$\Delta P=\frac{2\left(y-P_{s}\right)}{R}$

where $P_{F}$ and $R$ are the particle-induced surface pressure and the droplet radius, respectively.

By shrinkage of droplet and consequently reduction of distance between particles, repulsive interaction forces increase leading to the higher particle-induced surface pressure. Therefore, the pressure difference decreases overtime as shown in Fig.5. When the particle induces pressure is larger than surface tension ( $\mathrm{Ps}>\gamma$ ), deformation of droplet originates in locations with lower concentration of particles which are weaker against the particle-induced surface pressure [42, 43]. In other words, the buckling process starts when the energy stored in packed aggregate reaches a threshold value and conquers the total free energy of droplet including bulk free energy and interfacial energy $(\Delta \mathrm{P}=0)$ (Fig.5). The interplay between the shell energy and the opposing droplet surface tension energy determines the magnitude of deformation. The energy associated with an inversion of depth $\zeta$ can be expressed as $[44,45]$ :

$E_{e l}=c \chi \beta^{1 / 4}\left(\zeta / h_{R}\right)^{\frac{3}{2}}+P_{e x} \Delta V$

where

$\Delta V \propto-R \zeta^{2}$

where c, $\chi, \beta, P_{\text {ex }}$ and $\Delta \mathrm{V}$ are a dimensionless contact, bending rigidity, $F$ öppl-Kármán number, external pressure and the volume change due to the inversion, respectively. For large values of inversion depth, the second term of becomes dominant, and inversions can decrease their net energy by growing even deeper. The maximum value of inversion depth occurs when [44]:

$$
\begin{aligned}
& \chi \beta^{1 / 4}\left(\zeta^{1 / 2} / R^{3 / 2}\right) \sim P_{e x} R \zeta \rightarrow \\
& \frac{\zeta_{\max }}{R} \sim\left(\frac{\chi}{P_{\sigma \infty} R^{3}}\right)^{2} \sqrt{\beta}
\end{aligned}
$$

The eq. (16) indicates that for a particular pressure, inversions of depth become larger overtime until some other constraint such as self-contact avoids further growth of the inversion [44].

\begin{tabular}{|c|c|}
\hline \multicolumn{2}{|c|}{ Table1: simulation conditions } \\
\hline Rate of Evaporation $\left({ }^{K}\right)$ & $1.68 \times 10^{-6}\left(\mathrm{~m}^{2} / \mathrm{s}\right)$ \\
\hline Particle Diffusivity $\left(D_{\mathrm{pl}}\right)$ & $\left.5.95 \times 10^{-13} \mathrm{~m}^{2} / \mathrm{s}\right)$ \\
\hline $\begin{array}{c}\text { Peclet number } \\
\left.P e={ }^{K} / 8 D_{\text {pl }}\right)\end{array}$ & $3.53 \times 10^{5}$ \\
\hline Stiffness Parameter & $0.4 Y$ \\
\hline Droplet Initial radius $\left(R_{o}\right)$ & $10 \mu \mathrm{m}$ \\
\hline Particles radius $\left({ }^{F_{p}}\right)$ & $400 \mathrm{~nm}$ \\
\hline Number of Particles & 5000 \\
\hline
\end{tabular}

\begin{tabular}{|c|c|c|c|}
\hline \multicolumn{4}{|c|}{ Table2: properties of the materials used in the simulations. } \\
\hline & \multicolumn{3}{|c|}{ Properties } \\
\hline Material & $\begin{array}{c}\text { Density, } \\
K g / \mathrm{m}^{2}\end{array}$ & $\begin{array}{c}\text { Dynamic } \\
\text { viscosity, } \\
\mathrm{kg} \cdot \mathrm{m} / \mathrm{s}\end{array}$ & $\begin{array}{c}\text { Surface } \\
\text { tension, } \\
\mathrm{N} / \mathrm{m}\end{array}$ \\
\hline Ethanol & 789 & 0.00123 & 0.022 \\
\hline $\begin{array}{c}\text { Yttria } \\
\text { Stabilized } \\
\begin{array}{c}\text { Zirconia } \\
\text { (YSZ) }\end{array}\end{array}$ & 5560 & - & - \\
\hline
\end{tabular}




$$
\begin{gathered}
\mathrm{t}=0 \mu \mathrm{sec}, \\
\theta=0
\end{gathered}
$$

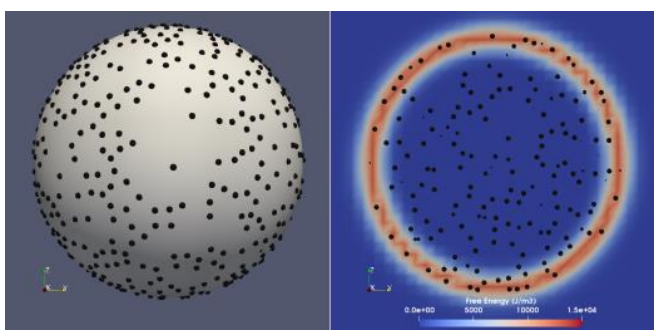

(a)

$$
\begin{gathered}
\mathrm{t}=56 \mu \mathrm{sec}, \\
\theta=0.233
\end{gathered}
$$

$\theta=0.483$

(Onset of

Buckling)

$\mathrm{t}=176 \mu \mathrm{sec}$,

$\theta=0.733$

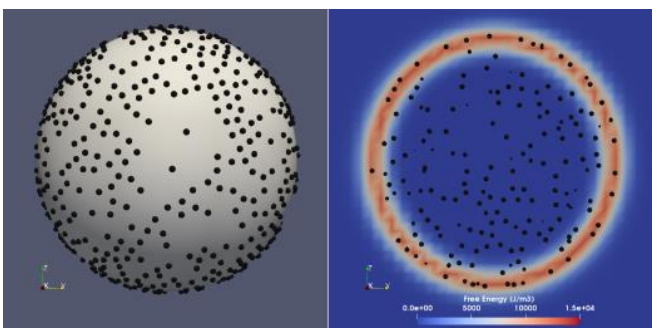

(b)

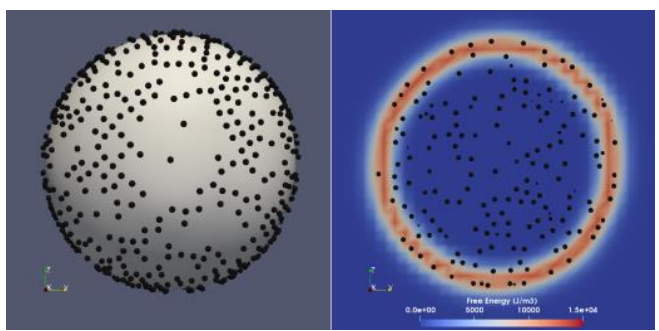

(c)

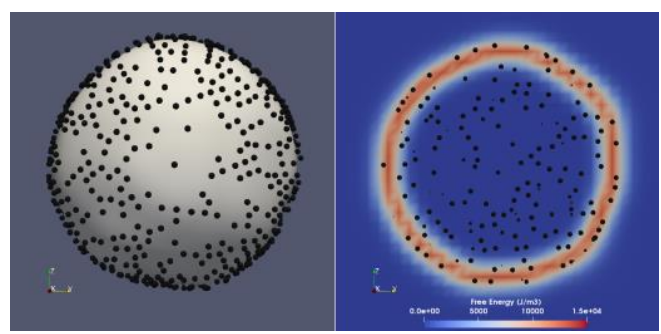

(d)

$\mathrm{t}=208 \mu \mathrm{sec}$,

$\theta=0.866$

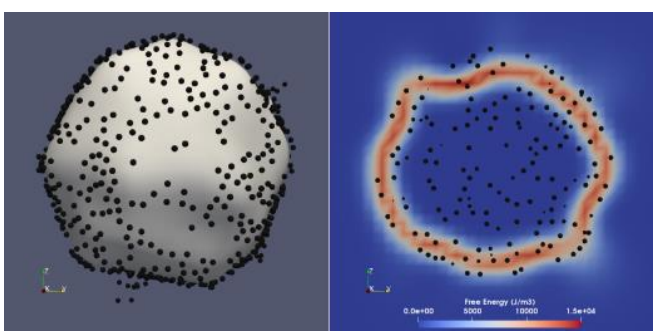

(e)

$\mathrm{t}=240 \mu \mathrm{sec}$,

$$
\theta=1
$$

\section{(f)}

Fig.3 Snapshot of numerical simulation (a) $\theta=0$ (b) $\theta=0.233$ (c) $\theta=0.483$ (d) $\theta=0.733$ (e) $\theta=0.866$ (f) $\theta=1(\theta=\mathrm{t} \tau, \tau$ is total time of buckling process $(240 \mu \mathrm{s}))$ Left images: spatial distribution of ethanol and solid particles. Right images: free energy contour.

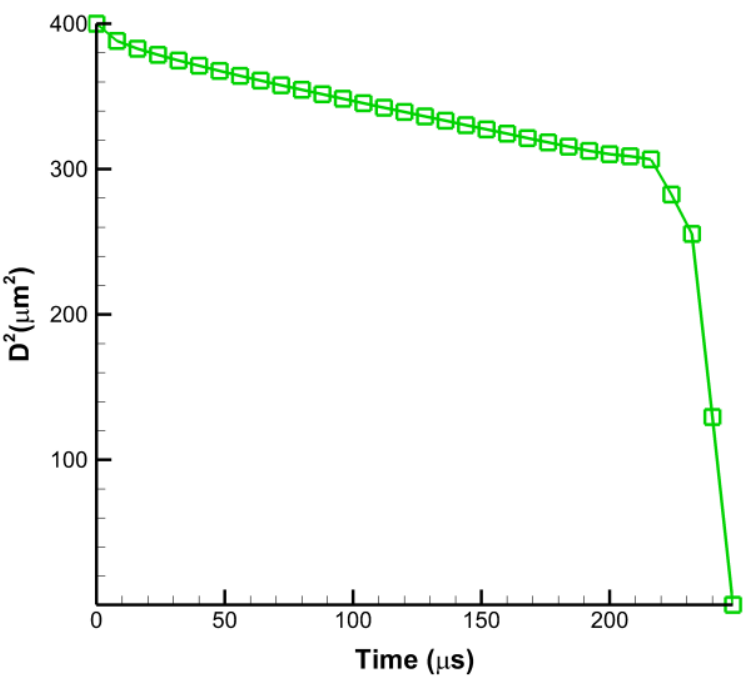

Fig.4 Time history of droplet diameter 


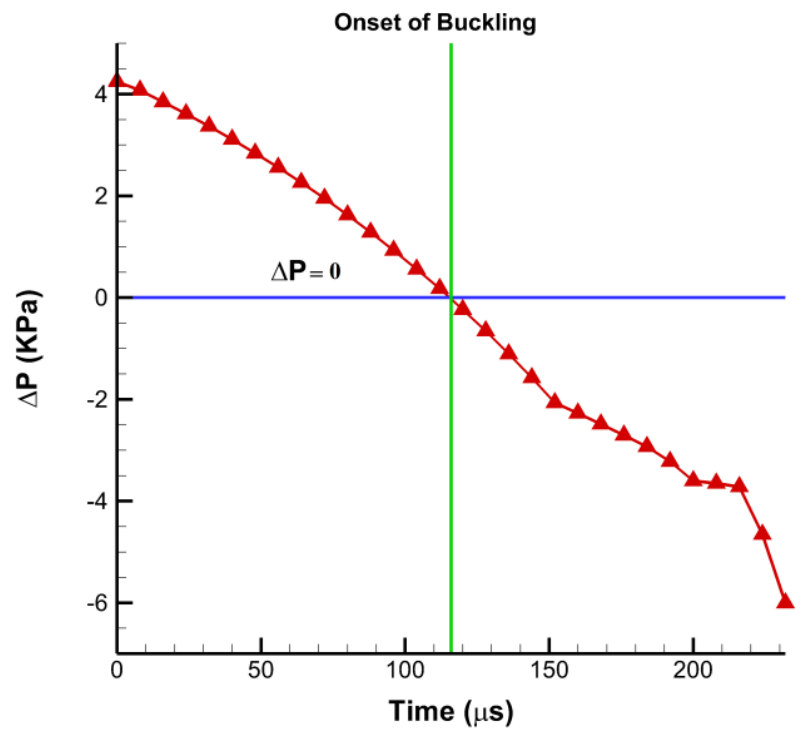

Fig.5 Time history of pressure difference between inside and outside of

droplet

\section{CONCLUSION}

In this work, a consistent numerical method had been developed to investigate the evaporation of suspension droplet in high rate evaporation processes. The developed model used a Eulerian-Lagrangian approach based on FIPI method for fluid and suspended solid particles, and the level set method to capture the droplet interface during evaporation. A linear model was developed to calculate the particle-particle interaction force which not only reduced the computation cost but also resulted in a stable simulation. The model can predict the successive stage of drying of a suspension droplet reported in the literature. The numerical results showed that the particleinduced surface pressure gradually increases during evaporation and reaches a critical value. At onset of buckling, the particle-induced surface pressure is equal to the droplet surface tension. From that point on, the droplet gradually buckles at locations with lower concentration.

\section{REFRENCES}

[1] J.-Y. Leong, B.-T. Tey, C.-P. Tan, E.-S. Chan, Nozzleless fabrication of oil-core biopolymeric microcapsules by the interfacial gelation of Pickering emulsion templates, ACS applied materials \& interfaces 7(30) (2015) 16169-16176.

[2] J.-Y. Leong, W.-H. Lam, K.-W. Ho, W.-P. Voo, M.F.-X. Lee, H.-P. Lim, S.-L. Lim, B.-T. Tey, D. Poncelet, E.-S. Chan, Advances in fabricating spherical alginate hydrogels with controlled particle designs by ionotropic gelation as encapsulation systems, Particuology 24 (2016) 44-60.

[3] W.W. Mwangi, K.-W. Ho, C.-W. Ooi, B.-T. Tey, E.-S. Chan, Facile method for forming ionically cross-linked chitosan microcapsules from Pickering emulsion templates, Food hydrocolloids 55 (2016) 26-33.

[4] Y. Yang, Z. Fang, X. Chen, W. Zhang, Y. Xie, Y. Chen, Z. Liu, W. Yuan, An overview of Pickering emulsions: solidparticle materials, classification, morphology, and applications, Frontiers in pharmacology 8 (2017) 287.

[5] V.Z. Beachley, M.T. Wolf, K. Sadtler, S.S. Manda, H. Jacobs, M.R. Blatchley, J.S. Bader, A. Pandey, D. Pardoll, J.H. Elisseeff, Tissue matrix arrays for high-throughput screening and systems analysis of cell function, Nature methods 12(12) (2015) 1197.

[6] W. Sempels, R. De Dier, H. Mizuno, J. Hofkens, J. Vermant, Auto-production of biosurfactants reverses the coffee ring effect in a bacterial system, Nature communications 4 (2013) 1757.

[7] F. Jabbari, M. Jadidi, R. Wuthrich, A. Dolatabadi, A numerical study of suspension injection in plasma-spraying process, Journal of Thermal Spray Technology 23(1-2) (2014) 3-13.

[8] M. Jadidi, M. Mousavi, S. Moghtadernejad, A. Dolatabadi, A three-dimensional analysis of the suspension plasma spray impinging on a flat substrate, Journal of Thermal Spray Technology 24(1-2) (2015) 11-23.

[9] S.C. Bradford, On the Theory of Gels, Biochemical Journal 12(4) (1918) 351.

[10] A. Saha, S. Seal, B. Cetegen, E. Jordan, A. Ozturk, S. Basu, Thermo-physical processes in cerium nitrate precursor droplets injected into high temperature plasma, Surface and Coatings Technology 203(15) (2009) 2081-2091.

[11] C. Handscomb, M. Kraft, A. Bayly, A new model for the drying of droplets containing suspended solids after shell formation, Chemical Engineering Science 64(2) (2009) 228246.

[12] M. Wozniak, G. Derkachov, K. Kolwas, J. Archer, T. Wojciechowski, D. Jakubczyk, M. Kolwas, Formation of highly ordered spherical aggregates from drying microdroplets of colloidal suspension, Langmuir 31(28) (2015) 7860-7868.

[13] M. Jaskulski, P. Wawrzyniak, I. Zbiciński, CFD model of particle agglomeration in spray drying, Drying Technology 33(15-16) (2015) 1971-1980.

[14] Z. Wang, T. Guo, L. Tian, Q. Xu, S. Zhan, J. Tu, Numerical simulation on circulation flow and mass transfer inside atmospheric water drops, Applied Thermal Engineering 118 (2017) 765-772.

[15] A. Farrokhpanah, T.W. Coyle, J. Mostaghimi, Numerical study of suspension plasma spraying, Journal of Thermal Spray Technology 26(1-2) (2017) 12-36.

[16] R. Kumaki, T. Moriya, T. Takahashi, An expression model of blood drying process based on SPH method, 2018 International Workshop on Advanced Image Technology (IWAIT), IEEE, 2018, pp. 1-4.

[17] H. Esmailzadeh, M. Passandideh-Fard, Numerical and experimental analysis of the fluid-structure interaction in presence of a hyperelastic body, Journal of Fluids Engineering 136(11) (2014) 111107. 
[18] Y. Yamada, M. Sakai, Lagrangian-Lagrangian simulations of solid-liquid flows in a bead mill, Powder technology 239 (2013) 105-114.

[19] A. Farrokhpanah, Applying contact angle to a twodimensional smoothed particle hydrodynamics (SPH) model on a graphics processing unit (GPU) platform, University of Toronto, 2012.

[20] D.J. Price, Modelling discontinuities and KelvinHelmholtz instabilities in SPH, Journal of Computational Physics 227(24) (2008) 10040-10057.

[21] J.P. Morris, Simulating surface tension with smoothed particle hydrodynamics, International journal for numerical methods in fluids 33(3) (2000) 333-353.

[22] H. Gotoh, H. Ikari, T. Memita, T. Sakai, Lagrangian particle method for simulation of wave overtopping on a vertical seawall, Coastal Engineering Journal 47(2-3) (2005) 157-181.

[23] G.-R. Liu, M.B. Liu, Smoothed particle hydrodynamics: a meshfree particle method, World scientific2003.

[24] S. Koshizuka, Y. Oka, Moving-particle semi-implicit method for fragmentation of incompressible fluid, Nuclear science and engineering 123(3) (1996) 421-434.

[25] D. Pfleger, S. Gomes, N. Gilbert, H.-G. Wagner, Hydrodynamic simulations of laboratory scale bubble columns fundamental studies of the Eulerian-Eulerian modelling approach, Chemical Engineering Science 54(21) (1999) 50915099.

[26] S.M. Javid, M. Passandideh-Fard, A. Faezian, M. Goharimanesh, Slug and bubble flows in a flat sheet ultrafiltration module: Experiments and numerical simulation, International Journal of Multiphase Flow 91 (2017) 39-50.

[27] R. Kuriakose, C. Anandharamakrishnan, Computational fluid dynamics (CFD) applications in spray drying of food products, Trends in Food Science \& Technology 21(8) (2010) 383-398.

[28] F. Vejahati, N. Mahinpey, N. Ellis, M.B. Nikoo, CFD simulation of gas-solid bubbling fluidized bed: a new method for adjusting drag law, The Canadian Journal of Chemical Engineering 87(1) (2009) 19-30.

[29] J.A. Sethian, P. Smereka, Level set methods for fluid interfaces, Annual review of fluid mechanics 35(1) (2003) 341-372.

[30] H. Montazeri, M. Bussmann, J. Mostaghimi, Accurate implementation of forcing terms for two-phase flows into SIMPLE algorithm, International Journal of Multiphase Flow 45 (2012) 40-52.

[31] M. Pasandideh - Fard, Y. Qiao, S. Chandra, J. Mostaghimi, Capillary effects during droplet impact on a solid surface, Physics of fluids 8(3) (1996) 650-659.

[32] S. Alavi, M. Passandideh-Fard, J. Mostaghimi, Simulation of semi-molten particle impacts including heat transfer and phase change, Journal of Thermal Spray Technology 21(6) (2012) 1278-1293.

[33] H. Montazeri, C. Ward, A balanced-force algorithm for two-phase flows, Journal of Computational Physics 257 (2014) 645-669.
[34] C. Gu, L. Botto, Direct calculation of anisotropic surface stresses during deformation of a particle-covered drop, Soft matter 12(3) (2016) 705-716.

[35] N. Samkhaniani, M. Ansari, Numerical simulation of bubble condensation using CF-VOF, Progress in Nuclear Energy 89 (2016) 120-131.

[36] C. Gu, L. Botto, Buckling vs. particle desorption in a particle-covered drop subject to compressive surface stresses: a simulation study, Soft matter 14(5) (2018) 711-724.

[37] P. Yue, C. Zhou, J.J. Feng, C.F. Ollivier-Gooch, H.H. Hu, Phase-field simulations of interfacial dynamics in viscoelastic fluids using finite elements with adaptive meshing, Journal of Computational Physics 219(1) (2006) 47-67.

[38] H. Zhu, Z. Zhou, R. Yang, A. Yu, Discrete particle simulation of particulate systems: theoretical developments, Chemical Engineering Science 62(13) (2007) 3378-3396.

[39] H.C. Hamaker, The London-van der Waals attraction between spherical particles, physica 4(10) (1937) 1058-1072.

[40] H. Zhuang, P. Lu, S.P. Lim, H.P. Lee, Study of the evaporation of colloidal suspension droplets with the quartz crystal microbalance, Langmuir 24(15) (2008) 8373-8378.

[41] F. Sicard, A. Striolo, Buckling in armored droplets, Nanoscale 9(25) (2017) 8567-8572.

[42] S.M. Javid, C. Moreau, J. Mostaghimi, Numerical Analysis of Buckling of a Single Suspension Droplet, Journal of Thermal Spray Technology 29(3) (2020) 344-357.

[43] S.M. Javid, J. Mostaghimi, C. Moreau, Evolution of Agglomerate Yttria Stabilized Zirconia Particles During Flight in Suspension Plasma Spray, The 2019 International Thermal Spray Conference and Exposition (ITSC), 2019.

[44] J. Paulose, D.R. Nelson, Buckling pathways in spherical shells with soft spots, Soft Matter 9(34) (2013) 8227-8245.

[45] A.V.e. Pogorelov, Bendings of surfaces and stability of shells, American Mathematical Soc. 1988. 